\title{
Which Nebulizer Position Should Be Avoided? An Extended Study of Aerosol Delivery and Ventilator Performance during Noninvasive Positive Pressure Ventilation
}

\author{
Yun Peng Bing Dai Chun-Xiang Hu Jia Su Wei Tan Hong-Wen Zhao \\ Jian Kang
}

Department of Respiratory Medicine, the First Affiliated Hospital of China Medical University, Shenyang, China

\section{Keywords \\ Noninvasive ventilation - Positive pressure ventilation . \\ Aerosol delivery $\cdot$ Simulation $\cdot$ Lung $\cdot$ Nebulizer position}

\begin{abstract}
Background: Research on the effect of nebulizer location on aerosol delivery during noninvasive ventilation has reached inconsistent conclusions. Objective: To investigate the effects of nebulizer position on aerosol delivery efficiency and ventilator performance during noninvasive ventilation. Methods: The Active Servo Lung 5000 respiratory simulation system (ASL5000) was used to simulate a COPD patient. The noninvasive ventilator was set to the spontaneous breathing mode. Six nebulizer positions, 2 exhalation valve types (single-arch exhalation port and whisper swivel), 4 combinations of inspiratory and expiratory pressure, and 2 respiratory rates were used. Results: Significant differences between nebulizer positions existed in aerosol delivery $(p<$ 0.05). Aerosol delivery efficiency was lower for nebulizer locations on either side of the exhalation valve and next to the ventilator outlet. When the nebulizer was located between the exhalation valve and the simulated lung, increased inspiratory pressure increased and increased expiratory pressure
\end{abstract}

decreased delivery efficiency (both $p<0.05$ ). When the nebulization device was located between the exhalation valve and the ventilator, no obvious trend was observed. Compared to baseline, nebulization lowered the air leakage volume displayed on the ventilator. There were no differences in ventilator performance between different nebulizer positions. Conclusions: The closer the nebulizer was to the exhalation valves or ventilator, the lower the aerosol delivery efficiency. Nebulizer position had little clinically significant effect on ventilator performance.

(c) 2017 S. Karger AG, Basel

\section{Introduction}

Noninvasive positive pressure ventilation is an effective method for treating patients with respiratory failure, it has widespread clinical applications, and indications for this treatment are increasing steadily [1-3]. Most patients receiving noninvasive ventilation for respiratory failure also require aerosol inhalation of drugs such as bronchodilators, glucocorticoids, mucolytics, and antibiotics [4]. Research has found aerosol inhalation therapy in conjunction with noninvasive ventilation to be a more

\section{KARGER}

(c) 2017 S. Karger AG, Basel

E-Mail karger@karger.com

www.karger.com/res 
effective method of drug delivery than conventional nebulization (in between noninvasive positive pressure ventilation sessions) [5].

Noninvasive positive pressure ventilation generally employs a single breathing circuit equipped with a passive exhalation valve that allows intentional leakage of air. There is a significant difference in the amount of air delivered into the breathing circuit before and after the exhalation valve and its leakage, and factors affecting aerosol inhalation efficiency during noninvasive ventilation are complex. The effect of the nebulizer position is particularly complicated [6]. While most current investigators advocate using a nebulizer position at the distal end of the breathing circuit, between the exhalation valve and the patient [7], some studies have found that a nebulizer position at the proximal end of the breathing circuit, between the exhalation valve and the ventilator, can increase aerosol inhalation efficiency $[8,9]$. This inconsistency may be related to the different distances between the nebulizer position and the exhalation valve used in the different studies, where too short a distance from the nebulizer to the exhalation valve may cause excessive leakage of the aerosol through the exhalation valve, and too long a distance from the nebulizer to the valve (that is, a position closer to the ventilator) may cause excessive loss of the aerosol in the breathing circuit or aerosol reflux inside the ventilator.

In the present study, we established 6 nebulizer positions by adding an extension tube or taking advantage of the connectors in the tubing without impairing the integrity of the breathing circuit (Fig. 1). We then conducted simulations to compare the effect of different nebulizer positions on aerosol delivery efficiency during noninvasive ventilation, using 2 different types of exhalation valve and different combinations of inspiratory and expiratory pressure and breathing rates. In addition, although in theory the bias flow of $2-10 \mathrm{~L} / \mathrm{min}$ caused by nebulization into a breathing circuit will affect ventilator performance [10], there are as yet no studies concerning possible changes in ventilator performance caused by different nebulizer positions. Therefore, we recorded, in the same study, the effect of nebulization and different nebulizer positions on ventilator performance.

\section{Materials and Methods}

\section{Simulated Lung Setup}

The Active Servo Lung 5000 respiratory simulation system (ASL5000; IngMar Medical, USA) is a precision breathing simulator. It is comprised of a piston moving inside a cylinder and is able to simulate the respiratory movements of patients with various illnesses. The simulated lung used the following parameters to simulate a COPD patient: compliance, $60 \mathrm{~mL} / \mathrm{cm} \mathrm{H}_{2} \mathrm{O}$; inhalation resistance, $10 \mathrm{~cm} \mathrm{H}_{2} \mathrm{O} / \mathrm{L} / \mathrm{s}$; expiratory resistance, $15 \mathrm{~cm}$ $\mathrm{H}_{2} \mathrm{O} / \mathrm{L} / \mathrm{s}$; maximum drop in inspiratory pressure, $-8 \mathrm{~cm} \mathrm{H}_{2} \mathrm{O}$. To simulate the profile of the negative pressure created by respiratory muscles, $5 \%$ of the respiratory cycle time was active inspiration, $3 \%$ was an end-inspiratory hold, and $15 \%$ was for the return of pressure to baseline. The breathing rate was set at either 10 or 20 times/min.

\section{Noninvasive Ventilator Setup}

The noninvasive ventilator (Flexo; Curative Medical, USA) was set to the spontaneous breathing mode with an increased pressure gradient of 1 , an inspiration sensitivity of 1 , and an expiration sensitivity of 1 . The breathing circuit (312107; Respironics, USA) was approximately $2.4 \mathrm{~m}$ in length, included neither a condensate collection cup nor humidifiers, and was connected to the simulated lung in order to simulate noninvasive ventilation. The inspiratory and expiratory pressures were set at 15/5, 25/5, 15/10, and $25 / 10 \mathrm{~cm} \mathrm{H}_{2} 0$. The exhalation valve used was either a singlearch exhalation port or a whisper swivel (both Respironics; for more detailed descriptions of the valves see [8]).

\section{Simulation of Nebulization}

For nebulization, $1 \mathrm{~mL}$ of a $0.5 \%$ salbutamol solution (Ventolin; GlaxoSmithKline, Australia) was diluted in $3 \mathrm{~mL}$ of normal saline, and the solution was placed in a small-volume jet nebulizer (1884; Teleflex, Mexico). The nebulizer was driven by a wall oxygen source (50 psi), and the flow meter (Pacific Medical, Taiwan) maintained the oxygen flow at $8 \mathrm{~L} / \mathrm{min}$. Six nebulizer positions were established by adding an extension tube or using the connectors in the tubing (Fig. 1). These locations are as follows: location 1 (near the simulated lung and $15 \mathrm{~cm}$ from the distal end of the exhalation valve), location 2 (near the distal end of the exhalation valve), location 3 (near the proximal end of the exhalation valve), location 4 ( $80 \mathrm{~cm}$ from the proximal end of the exhalation valves, that is, approximately the location of the condensate collection cup), location 5 (160 cm from the proximal end of the exhalation valves, that is, approximately the location of the humidifiers), and location 6 (at the ventilator outlet).

Three experiments were conducted under each set of experimental conditions. The aerosol particles were intercepted by a disposable filter (REF19212; Teleflex Medical, Malaysia) placed at the entrance to the simulated lung. A stopwatch was used to record the nebulization time, and nebulizations were considered finished when there was no visible evidence of nebulization for a period of $30 \mathrm{~s}$. The ventilator was switched off for at least $30 \mathrm{~s}$ after the end of the nebulization time. All experiments were performed by the same investigator.

\section{Aerosol Measurement}

Following each nebulization, the filter was washed using $10 \mathrm{~mL}$ normal saline to collect the aerosol. The filter was shaken using a vortex shaker (XW-80A; Huxi, Shanghai) for $1 \mathrm{~min}$ in order to fully mix the normal saline and salbutamol aerosol particles. The washing solution was placed in a 1-mL quartz glass cup, and a UV spectrophotometer was used to measure the absorbance of the solution at a wavelength of $276 \mathrm{~nm}$. Absorbance at this wavelength had a linear relationship with the concentration of the salbutamol 


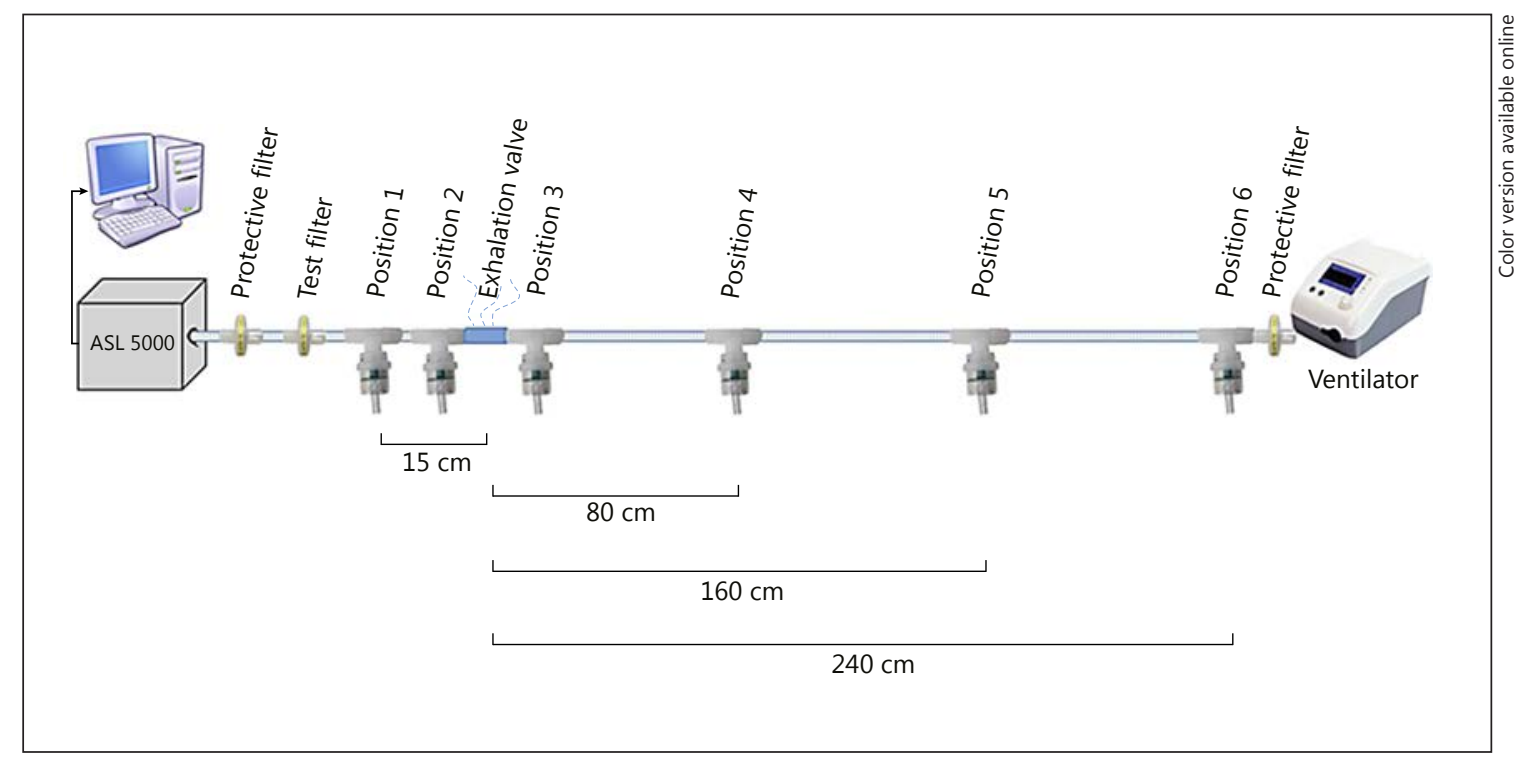

Fig. 1. Schematic diagram of experimental setup.

solution over the concentration range of 0 to $0.1 \mathrm{mg} / \mathrm{mL}$, and the slope of the standard curve was $0.1426\left(r^{2}=0.99\right)$. The standard curve was then used to calculate the corresponding salbutamol concentration and amount.

\section{Measurement of Ventilator Performance Parameters}

One minute after the start of each round of nebulization, the built-in software of the ASL5000 recorded inspiratory tidal volume, peak flow, peak inspiratory pressure, trigger time (the time needed from the start of inspiration in the simulated lung to the time the minimum airway pressure needed to trigger the ventilator is reached), and $\mathrm{T}_{90 \%}$ (the time needed to reach $90 \%$ of maximum pressure) for 10 continuous breathing cycles. It also recorded the tidal volume displayed by the ventilator during the 10 continuous cycles and the number of auto-triggering or missed trigger events during the entire nebulization process.

\section{Statistical Analysis}

Aerosol delivery efficiency and ventilator performance were presented as mean and standard deviation. One-way analysis of variance (ANOVA) with the Bonferroni post hoc test was performed to compare differences in nebulization efficiency between the 6 locations at each inspiratory pressure/expiratory pressure. Paired $t$ tests were performed to compare differences of ventilator performance between baseline and each nebulizer location. All analyses were stratified by exhalation valve type, respiratory rate, and inspiratory pressure/expiratory pressure. Statistical analyses were performed by IBM SPSS statistical software version 22 for Windows (IBM Corp., Armonk, NY, USA). Two-tailed $p<0.05$ indicated statistical significance for comparisons of nebulization efficiency. For ventilator performance, two-tailed $p<0.05$ and changes greater than $10 \%$ compared to baseline indicated statistical and clinical significance, respectively.

Nebulizer Position on Aerosol Delivery Efficiency

\section{Results}

High and Low Aerosol Delivery Efficiency

Aerosol delivery efficiency at different nebulizer locations under different inspiratory pressures, expiratory pressures, and respiratory rates is shown using the single arch exhalation port (Fig. 2) and the whisper swivel exhalation port (Fig. 3).

Aerosol delivery efficiency in different nebulizer locations ranged from 3.3 to $35.2 \%$, and the locations in which these extremes were seen varied ( 3 lowest deliveries at location 3 and 1 at location 6, 2 highest deliveries at location 1 and 1 at location 4). In individual protocols, the largest difference between nebulizer locations in efficiency was about $50 \%$. This $50 \%$ difference was seen between nebulizer locations 1 and 6 for both the single arch and whisper valve exhalation ports when the respiration rate was 20 times/min, inspiratory pressure $25 \mathrm{~cm} \mathrm{H}_{2} \mathrm{O}$, and expiratory pressure $5 \mathrm{~cm} \mathrm{H}_{2} \mathrm{O}$. The smallest difference in aerosol delivery was about $10 \%$, and this was seen between nebulizer locations 3 and 5 for the single arch exhalation port and locations 1 and 3 for the whisper swivel exhalation port, with expiratory pressures of 5 and 10 $\mathrm{cm} \mathrm{H}_{2} \mathrm{O}$, respectively, an inspiratory pressure of $25 \mathrm{~cm}$ $\mathrm{H}_{2} \mathrm{O}$, and a respiratory rate of 10 times $/ \mathrm{min}$. 

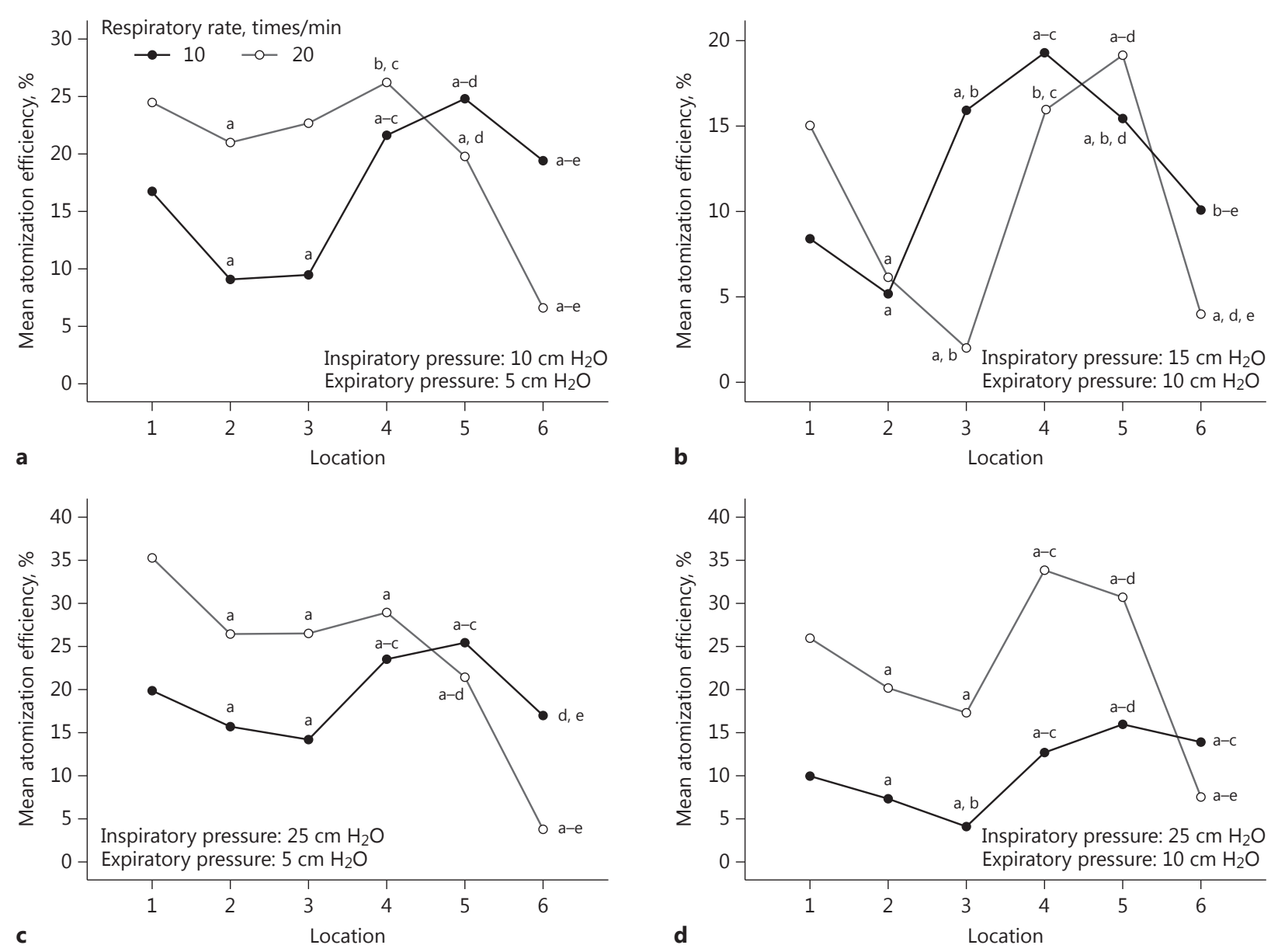

Fig. 2. a-d Comparisons between different locations in nebulization efficiency for the exhalation valve type of single-arch exhalation port. ${ }^{a}$ Significantly different from location $1 ;{ }^{b}$ significantly different from location $2 ;^{c}$ significantly different from location 3 ; $^{\mathrm{d}}$ significantly different from location 4 ; ${ }^{\mathrm{e}}$ significantly different from location 5.

\section{Aerosol Delivery at Nebulizer Locations between the Lung and the Exhalation Port}

When the nebulizer was located between the exhalation port and the simulated lung, aerosol delivery was significantly more efficient $(p<0.05)$ when the nebulizer was placed near the lung (location 1) than when placed near the exhalation port (location 2). In addition, when the nebulizer was located in this section of the respiratory circuit, an increase in breathing rate decreased aerosol delivery efficiency. Also, an increase in inspiratory pressure increased aerosol delivery efficiency while an increase in expiratory pressure decreased aerosol delivery efficiency (both $\mathrm{P}<0.05$ ). This trend was observed under all conditions tested.

\section{Aerosol Delivery at Nebulizer Locations between the Exhalation Port and the Ventilator}

When the nebulizer was placed between the exhalation port and the ventilator, aerosol delivery was most efficient in the middle section of this region (locations 4 and 5), except in one protocol (whisper swivel, inspiratory pressure $25 \mathrm{~cm} \mathrm{H}_{2} \mathrm{O}$, expiratory pressure $10 \mathrm{~cm} \mathrm{H}_{2} \mathrm{O}$, respiratory rate 10 times/min) where the highest delivery (only about 7\%) was seen at location 6 , the location closest to the ventilator outlet. Increases in breathing rate, inspiratory pressure, and expiratory pressure had mixed effects on aerosol delivery in the exhalation-port-to-ventilator section of the breathing circuit, and no clear trend was observed. 

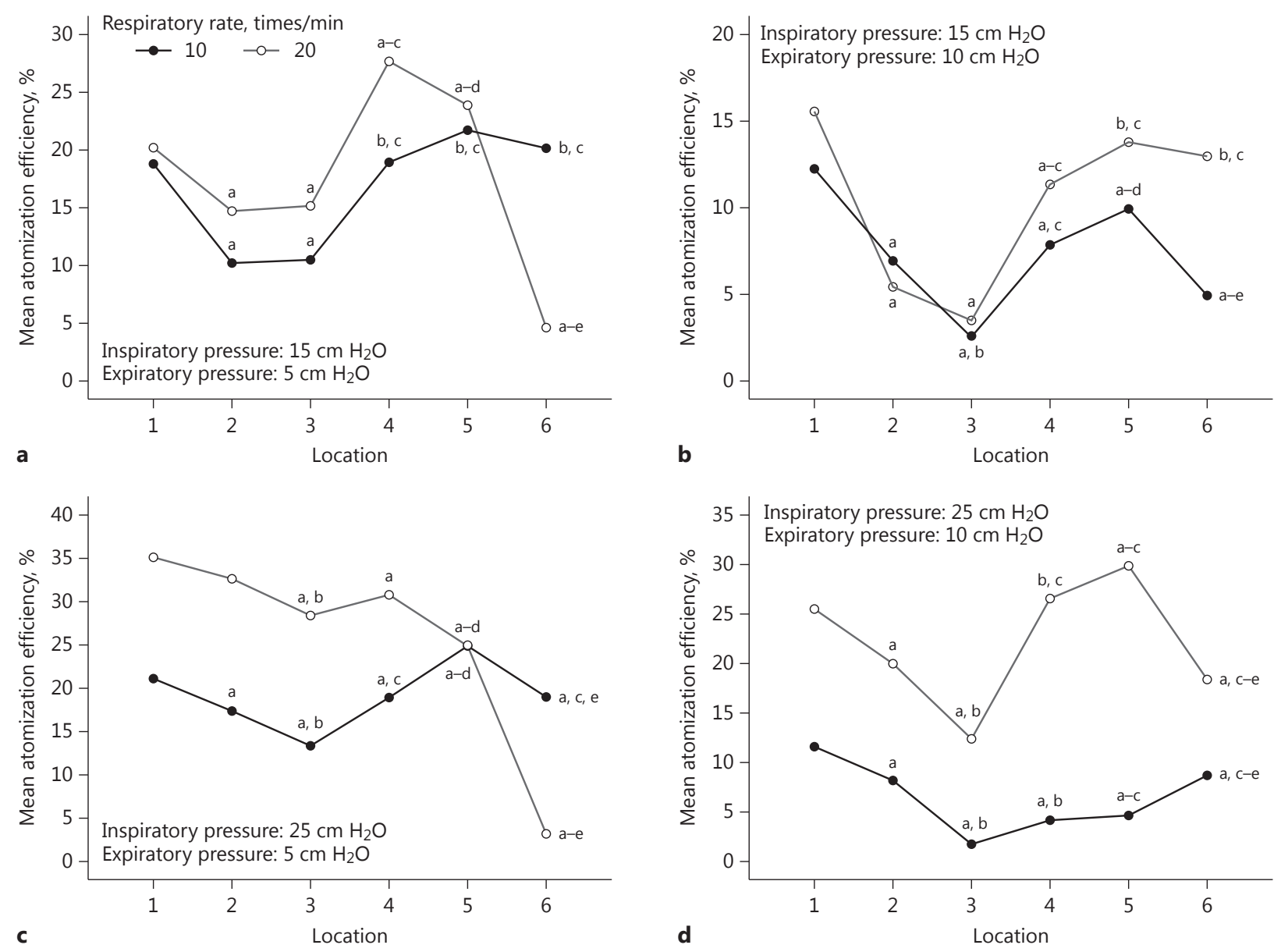

Fig. 3. a-d Comparisons between different locations in nebulization efficiency for the exhalation valve type of whisper swivel. ${ }^{\text {a }}$ Significantly different from location $1 ;{ }^{b}$ significantly different from location $2 ;^{c}$ significantly different from location $3{ }^{\mathrm{d}}$ significantly different from location $4{ }^{\mathrm{e}}$ significantly different from location 5.

In summary, aerosol delivery was relatively less efficient when the nebulizer was positioned next to the exhalation port on either side (locations 3 and 4 ) or next to the ventilator outlet (location 6). Increases in inspiratory pressure, expiratory pressure, and breathing rate respectively increased, decreased, and decreased aerosol delivery in the exhalation-port-to-lung section of the respiratory circuit, and had mixed effects in the exhalation-valeto-ventilator section.

\section{Nebulizer Location and Nebulization Effects on \\ Ventilator Performance}

Nebulization lowered the air leakage volume displayed on the ventilator compared to the baseline value.
Under certain experimental conditions (respiratory rate 10 times/min or single-arch exhalation port), nebulization significantly affected the ventilator triggering performance (time to trigger and trigger pressure) and increased the tidal volume displayed on the ventilator compared to baseline values. Nebulization did not affect peak inspiratory pressure, inspiratory time, tidal volume displayed on the simulated lung, or peak inspiratory flow.

The position of the nebulizer had no significant effect on ventilator performance (see online suppl. tables 1-8; see www.karger.com/doi/10.1159/000481868 for all online suppl. material). Aerosol nebulization time was $13.6 \pm 1.8 \mathrm{~min}$. No instances of false triggering or sponta- 
Table 1. Summary of investigations of the effect of aerosol inhalation location on aerosol delivery efficiency in past research

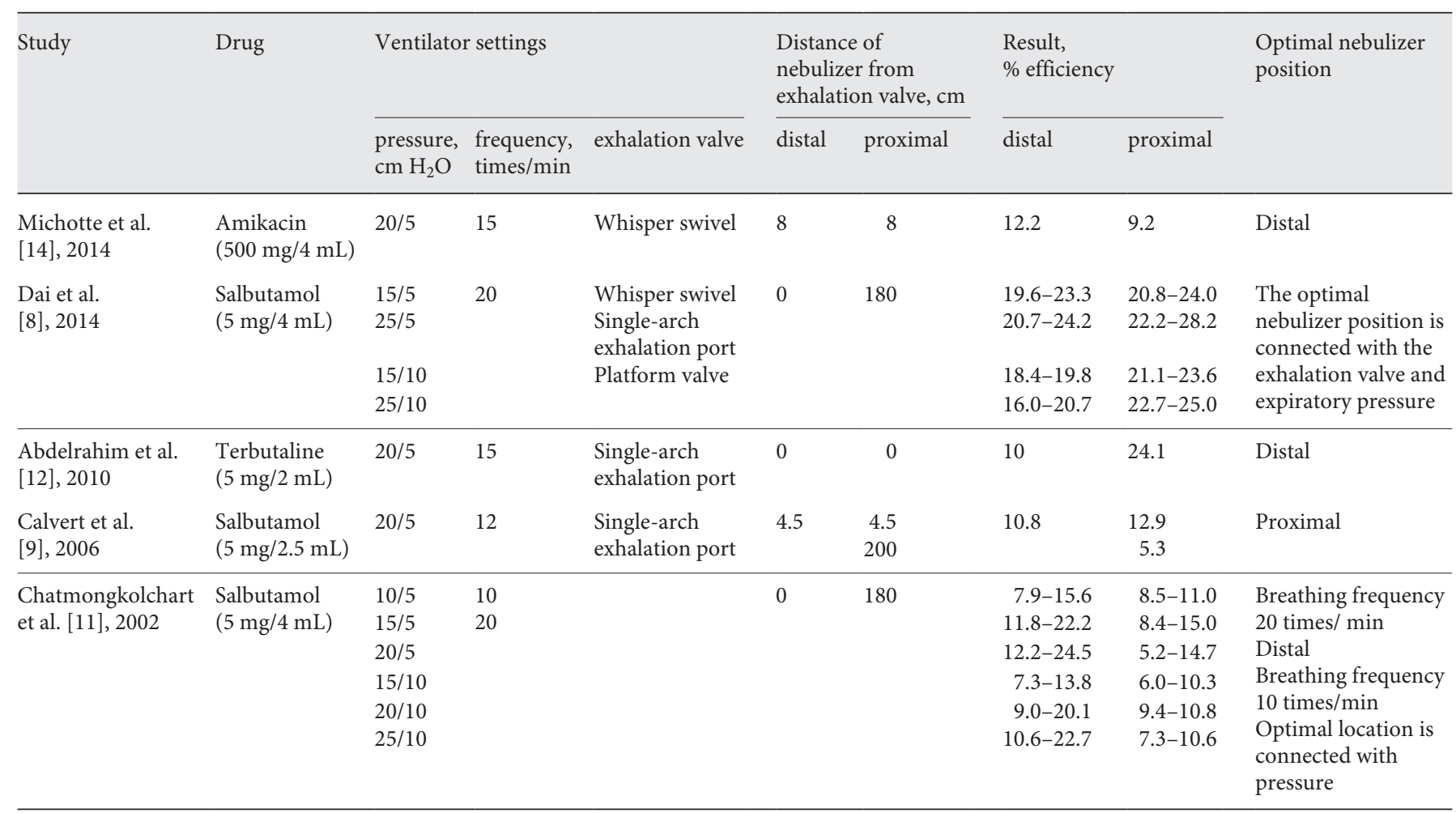

neous triggering (autotriggering or missed triggers) of the ventilator were observed during the nebulization process in any of the protocols.

\section{Discussion}

In the current study, nebulizer position had a significant and consistent effect on aerosol delivery efficiency: the closer the nebulizer was to the exhalation valves or ventilator, the lower the aerosol delivery efficiency. Increased inspiratory pressure increased delivery efficiency, and increased expiratory pressure decreased delivery efficiency. Respiratory rate had negative effects on aerosol delivery in the distal position (between the exhalation valve and the simulated lung), but mixed effects in the proximal position. Nebulizer position and nebulization process had little clinically significant effect on ventilator performance.

\section{Proximal versus Distal Nebulization}

Of 5 previous studies $[8,9,11-13]$ (Table 1$), 1$ reported that the proximal position gave higher delivery [9], 2 reported that the distal position had higher delivery [12,
13], and the other 2 studies gave inconsistent results [8, 11]. These discrepancies may be partly explained by differences in the distance between the nebulizer position and the exhalation valve in different studies, as well as the differences in experimental conditions used (Table 1). Chatmongkolchart et al. [11] found that while a distal position produced higher aerosol delivery efficiency at a breathing rate of 20 times/min, when the breathing rate was 10 times/min the proximal position was superior to the distal position. Our previous research found that when a single-arch exhalation port was used, and expiratory pressure was $5 \mathrm{cmH}_{2} \mathrm{O}$, the distal position had a higher delivery efficiency, but when expiratory pressure was increased to $10 \mathrm{cmH}_{2} \mathrm{O}$, the result was the opposite [8]. Also, when either a platform breather valve or a whisper swivel breather valve was used, the proximal position had the higher delivery efficiency [8]. As a result, we cannot derive an optimal nebulizer position under all conditions.

Positive Effects versus Negative Effects. When the nebulizer is at the proximal position, the breathing tube before or after the nebulization device performs a function as an aerosol spacer, and can therefore have positive effects, improving aerosol delivery efficiency. The presence of the 
exhalation valve causes aerosol to be expelled from the breathing circuit, and reflux inside the ventilator may also cause losses of aerosol. These negative effects will reduce aerosol delivery efficiency. Under some experimental conditions, when the positive effects are greater than the negative effects, one nebulizer position may be superior to another. In our study, nebulization positions 4 and 5 yielded relatively better delivery efficiency than positions 3 and 6 through enhancement of the above positive effects and a reduction of the accompanying negative effects.

Our study found that when the nebulizer is at the distal position, the delivery efficiency at location 1 is better than that at location 2. Location 1 is $15 \mathrm{~cm}$ from location 2 by adding an extension tube, which acts as an aerosol spacer and reduces aerosol to be expelled from the exhalation valve directly. Other studies have made similar findings. When aerosol inhalation is used during invasive ventilation, aerosol delivery efficiency is higher when the nebulization device is located $15 \mathrm{~cm}$ from the $\mathrm{Y}$-shaped tube [14]. When aerosol inhalation is used during spontaneous breathing, no matter what type of nebulization device is used, the addition of an extension tube and aerosol storage bag, which act as an aerosol spacer, can significantly increase delivery efficiency [15].

Concern of $\mathrm{CO}_{2}$ Rebreathing. When a $15-\mathrm{cm}$ extension tube is added at the distal end, this is equivalent to adding roughly $300 \mathrm{~mL}$ of dead space to the breathing circuit, which can increase $\mathrm{CO}_{2}$ rebreathing. The amount of $\mathrm{CO}_{2}$ rebreathing has been shown to be correlated with the amount of effective dead space, but is not entirely linked with the internal volume in the interface [16]. The inner cavity of a nasal-oral mask has a volume of approximately $100-200 \mathrm{~mL}$, and that of a full-face mask has a volume of approximately $900 \mathrm{~mL}$. In vitro studies have found that use of a full-face mask can increase $\mathrm{CO}_{2}$ rebreathing to a maximum of $3 \mathrm{~mL}$ during each breath [17]. Likewise, clinical research has reported that full-face masks and nasal-oral masks have similar influence on $\mathrm{CO}_{2}$ rebreathing [18]. $\mathrm{CO}_{2}$ rebreathing is also connected with factors such as the type and location of the exhalation valve, as well as with the inspiratory and expiratory pressure [19]. Hence, adding a $15-\mathrm{cm}$ extension tube at the distal position may have only an extremely small effect on $\mathrm{CO}_{2}$ rebreathing.

Inspiratory Pressure, Expiratory Pressure, and Breathing Rate. In our study, increasing inspiration pressure increased and increasing expiration pressure decreased aerosol delivery in the distal position (exhalation-portto-lung section) and had mixed effects on the proximal position. Others have reported similar results $[8,11]$. In our study, an increase in breathing rate decreased aerosol delivery in the distal position and had mixed effects in the proximal position. Chatmongkolchart et al. [11] found that while a nebulizer position near the lung (distal to the ventilator) produced the best aerosol delivery efficiency at a breathing rate of 20 times/min, when the breathing rate was 10 times/min, a nebulizer position near the ventilator was superior to a location near the lung (distal to the ventilator). So, the combination of position with other factors that influence nebulization efficiency is extremely complex, and factors such as exhalation valve, inspiratory pressure, expiratory pressure, and breathing rate have interactive effects with each other and with the nebulizer position.

\section{Ventilator Performance}

During invasive ventilation, nebulization can have a large impact on the patient's tidal volume and on patientventilator synchronization [20]. As a consequence, it may be necessary to modify the ventilator's alarm range or appropriately adjust the mechanical ventilation mode and parameters when performing aerosol inhalation [21]. Noninvasive positive pressure ventilation imposes a greater demand for patient-ventilator synchronization than invasive ventilation, and a bias flow of $2-10 \mathrm{~L} / \mathrm{min}$ in the breathing circuit during aerosol inhalation may, in theory, affect patient-ventilator synchronization. Past research, relying solely on autotriggering or missed triggers as indicators of ventilator performance $[8,11]$, found that nebulization does not have a significant influence on ventilator performance. Our study used additional indicators, including triggering, control, and cycles, to perform a more comprehensive assessment, and found that nebulization reduces the leakage displayed on the ventilator and in most cases has no clinically significant effect on ventilator performance.

Because of the significant difference in the amount of airflow before and after the exhalation valve, different nebulizer positions relative to the exhalation valve position may have different effects on ventilator performance. However, in our study, there were no significant differences between different nebulizer positions on ventilator performance. This may be because noninvasive ventilators have excellent leakage compensation functions. Regardless of the nebulizer position, the additional increase in airflow will be equivalent to a reduction in leakage, and the leakage displayed by the ventilator will be reduced. Therefore, the ventilator will then automatically recalculate a baseline, and there will be no significant impact on patient-ventilator synchronization. 


\section{Drug Delivery}

Aerosol delivery reported in other studies has also been lower than that found in our study $[8,11,13]$, perhaps because of a suboptimal selection of nebulizer location in previous research or because a shorter nebulization time (10 $\mathrm{min})$ was used than the time used in our study (approx. $13 \mathrm{~min}$ ). Aerosolized drugs are currently not limited to bronchodilators, but also include glucocorticoids, mucolytics, and antibiotics. In view of the value of many of these drugs, it is necessary to prolong nebulization time in order to minimize the amount of residual drug solution [4]. The factor that ultimately determines the clinical effectiveness of aerosol inhalation is the aerosol deposition rate, which is affected by the diameter of the aerosol particles and the delivery efficiency. The conclusions of this study will consequently await verification from clinical research and study of the aerodynamic diameter of aerosol particles.

\section{Clinical Perspective}

Noninvasive positive pressure ventilation in patients with chronic lung disease or respiratory disease has been found to be associated with improved outcomes such as dyspnea and reduced intubation [22, 23]. Although optimization of nebulizer position can significantly enhance the effectiveness of nebulization during noninvasive ventilation, the combination of position with other factors that influence nebulization efficiency is extremely complex, because factors such as type of exhalation valve, inspiratory pressure, expiratory pressure, and breathing rate have interactive effects with each other and with nebulizer position. Hence, physicians should focus on what position to avoid rather than on what is the best position.

However, in clinical practice, factors such as inspiratory pressure, expiratory pressure, and breathing rate are generally determined by the patient's condition, while the nebulization position may be chosen by medical personnel subjectively making informed choices. Therefore, we believe the results of this study on the effect of nebulizer position have clinical value in connection with optimization of aerosol inhalation during noninvasive ventilation.

To conclude, regardless of whether the nebulizer position is at the proximal or the distal end of the ventilator, the closer it is to the exhalation valve or the ventilator, the lower the aerosol delivery efficiency. In addition, unlike nebulization during invasive ventilation, nebulization has little influence on ventilator performance during noninvasive ventilation, regardless of nebulizer position.

\section{Acknowledgment}

The study was supported by the Chinese National Natural Science Foundation (grant No. 81400061) and the Science and Technology Planning Project of Shenyang (grant No. F15-139-9-22).

\section{Financial Disclosure and Conflicts of Interest}

The authors declare that no conflicts of interest exist.

\section{References}

1 Kelly CR, Higgins AR, Chandra S: Videos in clinical medicine. Noninvasive positive-pressure ventilation. N Engl J Med 2015;372:e30.

2 Davidson C, Banham S, Elliott M, Kennedy D, Gelder C, Glossop A, Church C, CreaghBrown B, Dodd J, Felton T, Foëx B, Mansfield L, McDonnell L, Parker R, Patterson C, Sovani M, Thomas L: British Thoracic Society/Intensive Care Society Guideline for the ventilatory management of acute hypercapnic respiratory failure in adults. BMJ Open Respir Res 2016;3:e000133.

3 Allison MG, Winter ME: Noninvasive ventilation for the emergency physician. Emerg Med Clin North Am 2016;34:51-62.
4 Ehrmann S, Roche-Campo F, Bodet-Contentin, Razazi K, Dugernier J, Trenado-Alvarez J, Donzeau A, Vermeulen F, Thévoz D, Papanikolaou M, Edelson A, Yoshido HL, Piquilloud L, Lakhal K, Lopes C, Vicent C, Desachy A, Apiou-Sbirlea G, Isabey D, Brochard L; Reva Research Network, AT@ICU Study Group: Aerosol therapy in intensive and intermediate care units: prospective observation of 2,808 critically ill patients. Intensive Care Med 2016;42:192-201.

5 Ari A, Fink JB: Differential medical aerosol device and interface selection in patients during spontaneous, conventional mechanical and noninvasive ventilation. J Aerosol Med Pulm Drug Deliv 2016;29:95-106.

6 Hess DR: Aerosol therapy during noninvasive ventilation or high-flow nasal cannula. Respir Care 2015;60:880-891; discussion 891-893.
7 Fink JB: Aerosol drug therapy; in: Egan's Fundamentals of Respiratory Care, ed 10. St. Louis, Elsevier, 2013, pp 844-886.

8 Dai B, Kang J, Sun LF, Tan W, Zhao HW: Influence of exhalation valve and nebulizer position on albuterol delivery during noninvasive positive pressure ventilation. I Aerosol Med Pulm Drug Deliv 2014;27:125-132.

9 Calvert LD, Jackson JM, White JA, Barry PW, Kinnear WJ, O'Callaghan C: Enhanced delivery of nebulized salbutamol during non-invasive ventilation. J Pharm Pharmacol 2006;58: 1553-1557.

10 Ari A, Fink JB, Dhand R: Inhalation therapy in patients receiving mechanical ventilation: an update. J Aerosol Med Pulm Drug Deliv 2012;25:319-332. 
11 Chatmongkolchart S, Schettino GP, Dillman C, Kacmarek RM, Hess DR: In vitro, evaluation of aerosol bronchodilator delivery during noninvasive positive pressure ventilation: effect of ventilator settings and nebulizer position. Crit Care Med 2002;30:2515-2519.

12 Abdelrahim ME, Plant P, Chrystyn H: In-vitro characterisation of the nebulized dose during non-invasive ventilation. J Pharm Pharmacol 2010;62:966-972.

13 Michotte JB, Jossen E, Roeseler J, Liistro G, Reychler G: In vitro comparison of five nebulizers during noninvasive ventilation: analysis of inhaled and lost doses. J Aerosol Med Pulm Drug Deliv 2014;27:430-440.

14 Fink J, Ari A: Aerosol delivery to intubated patients. Expert Opin Drug Deliv 2013;10: 1077-1093.

15 Ari A, Restrepo RD; American Association for Respiratory Care: Aerosol delivery device selection for spontaneously breathing patients: 2012. Respir Care 2012;57:613-626.
16 Fodil R, Lellouche F, Mancebo J, Sbirlea-Apiou G, Isabey D, Brochard L, Louis B: Comparison of patient-ventilator interfaces based on their computerized effective dead space. Intensive Care Med 2011;37:257-262.

17 Schettino GP, Chatmongkolchart S, Hess DR, Kacmarek RM: Position of exhalation port and mask design affect $\mathrm{CO}_{2}$ rebreathing during noninvasive positive pressure ventilation. Crit Care Med 2003;31:2178-2182.

18 Ozsancak A, Sidhom SS, Liesching TN, Howard W, Hill NS: Evaluation of the total face mask for noninvasive ventilation to treat acute respiratory failure. Chest 2011;139: 1034-1041.
19 Medrinal C, Prieur G, Contal O, Villiot-Danger E, Doyle C, Reychler G, Quieffin J: Noninvasive ventilation: evaluation of $\mathrm{CO}_{2}$ washout by intentional leaking in three recent oronasal masks. A pilot study. Minerva Anestesiol 2015;81:526-532.

20 Ehrmann S, Lyazidi A, Louis B, Isabey D, Le Pennec D, Brochard L, Apiou-Sbirlea G: Ventilator-integrated jet nebulization systems: tidal volume control and efficiency of synchronization. Respir Care 2014;59:15081516.

21 Ari A: Aerosol therapy in pulmonary critical care. Respir Care 2015;60:858-874.

22 Dreher M, Ekkernkamp E, Schmoor C, Schoenheit-Kenn U, Winterkamp S, Kenn K: Pulmonary rehabilitation and noninvasive ventilation in patients with hypercapnic interstitial lung disease. Respiration 2015;89: 208-213.

23 Liu YJ, Zhao J, Tang H: Non-invasive ventilation in acute respiratory failure: a meta-analysis. Clin Med (Lond) 2016;16:514-523. 